\title{
Numerical Simulation of the Damage Behavior of a Concrete Beam with an Anisotropic Damage Model
}

\author{
Y. C. Long $^{\mathrm{a}, \mathrm{b}, 1}$ and C. T. Yu $\mathbf{u}^{\mathrm{b}}$ \\ a Key Laboratory of New Technology for Construction of Cities in Mountain Area, Chongqing \\ University, Chongqing, China \\ b School of Civil Engineering, Chongqing University, Chongqing, China \\ 1 longyc02@cqu.edu.cn
}

An anisotropic damage model was proposed to describe the nonlinear behavior of concrete beams under monotonic and cyclic loading. The hysteresis effect of concrete is approximately modeled by employing nonlinear loading/linear reloading stress paths in the model, which was implemented into ABAQUS. Linear, bilinear, exponential and Reinhardt strain softening functions are introduced to investigate their influence on accuracy of calculations. The load-deflection responses obtained by the damage model reflect the damage-induced nonlinear behavior of concrete beams, results are comparable with the test data. The strain softening functions significantly affect simulation accuracy, and the responses obtained by the Reinhardt function are in the best agreement with experimental ressults. The numerical data under cyclic loading are consistent with those obtained in the experiment, characterizing the degradation of stiffness and hysteresis effect of concrete beams. It is concluded that the anisotropic damage model can be used to simulate the nonlinear behavior of concrete structures.

Keywords: concrete structure, anisotropic damage model, hysteresis effect, numerical simulation, cyclic loading.

Introduction. Numerical simulation is an important method to investigate the damage and fracture mechanisms of the concrete structure. One of the critical issue for numerical simulation is to develop a constitutive model of concrete which represents its nonlinear behavior. The continuum damage mechanics theory (CDMT) was by far the most popular theory for developing a constitutive model of concrete.

Based on the assumption of isotropic damage, Mazars et al. [1, 2] and Papa [3] developed damage models of concrete by utilizing scalar damage variable to represent the degradation of stiffness, which established the fundamental framework of continuum damage mechanics. Cervera et al. [4] and Richard et al. [5] introduced weighted damage evolution rules into the scalar damage model to model the stiffness recovery effect resulting from the closing of microcracks upon load reversals. Cervera et al. [4] and Haussler-Combe and Kuhn [6] proposed rate-dependent damage models within the framework of CDMT, which evolve from the fundamental rate-independent scalar damage models via an additional viscoelastic contribution, to take into account the strain-rate sensitivity of concrete. Desprez et al. [7] formulated an isotropic damage model for FRP-confined concrete and analyzed the nonlinear response of FRP-confined concrete column under axial and flexural loads. Although those scalar damage models didn't account for the anisotropic features of concrete, they were widely used in the nonlinear analyses of concrete structures because of their high efficiency.

Second- or fourth-order damage tensor was employed by Papa and Taliercio [8], Alliche [9], Lu et al. [10], Badel et al. [11], and Francois [12], within the fundamental framework of continuum damage mechanics, to model the anisotropic properties of concrete damage and develop anisotropic damage models. The above models were applied to the analyses of damage and failure prediction of concrete structures subjected to cyclic 
and fatigue loads. $\mathrm{Wu}$ and $\mathrm{Xu}$ [13] introduced thermodynamically consistent projection operators into the damage models employing the spectral decomposition of the stress and strain tensor, which guarantees the fulfillment of energy conservation under arbitrary load history. Based on the concept of thermodynamics, Ngo et al. [14] proposed an anisotropic damage model for thermo-mechanical coupling and modeled the nonlinear response of concrete members under thermal and mechanical loading. Hariri-Ardebili and Mirzabozorg [15] employed anisotropic damage mechanics approach to model the cracking behavior in mass concrete and then conducted a numerically seismic assessment of the arch-reservoirfoundation system. Compared to the isotropic damage models, the governing equations of the anisotropic ones were much more complicated. Most of these models, therefore, employed linear paths to represent unloading/reloading behavior to obtain high numerical efficiency. They might get a closer result, when the hysteretic behavior could be modeled, to the realistic response of the concrete structure.

Long and He [16] developed an anisotropic damage model in which nonlinear unloading/linear reloading stress paths were employed to model the hysteretic behavior. This model was implemented into the finite element analysis (FEA) program ABAQUS [17] by programming its constitutive relation in user subroutine UMAT [18]. Although this model could reflect the stiffness degradation and hysteretic response of concrete sample under uniaxial tension or compression, its applicability to the concrete structure should be demonstrated.

1. Fundamental Governing Equations. There are three granted assumptions in Long's model [16]. First, the strain can be, for any arbitrary material point, decomposed into elastic and inelastic parts. Then, the material axes are mutually orthogonal and parallel to the principal directions of the stress tensor. Last, the damage relations are defined regarding stress and inelastic strain corresponding to the rotating material axes with the assumption that the damage is decoupled. According to these assumptions, the governing equations of the model are as follows:

$$
\sigma=\mathbf{D} \varepsilon
$$

where $\sigma$ and $\varepsilon$ denote the stress and strain tensor, respectively, and $\mathbf{D}$ is the constitutive matrix representing the stress-strain relation and given as

$$
\mathbf{D}=\left(\mathbf{E}_{0}^{-1}+\mathbf{T}_{r} \mathbf{G T}_{t}^{T}\right)^{-1}
$$

in which $\mathbf{E}_{0}$ is the linear elastic stiffness matrix, and $\mathbf{T}_{r}$ is the transformation matrix of strain from global coordinates to the local one defined by the rotating material axes. $\mathbf{G}$ stands for the damage matrix corresponding to flexibility in local coordinates and is defined as

$$
\mathbf{G}=\left|\begin{array}{ccc}
g_{1}\left(e_{1}^{i n}\right) & 0 & 0 \\
0 & g_{2}\left(e_{2}^{i n}\right) & 0 \\
0 & 0 & g_{3}\left(e_{3}^{i n}\right)
\end{array}\right|,
$$

where $g_{i}\left(e_{i}^{i n}\right)$ and $e_{i}^{i n}$ denote the secant flexibility and inelastic strain on damage axis $i=1,2,3$. Because the above-mentioned governing equations are nonlinear, the linearization method should be utilized to obtain the constitutive relation in the incremental form that can be referred to work [16].

As shown in Fig. 1, stress paths subjected to cyclic loading comprises the loading path, unloading path, reloading path, partial unloading path and partial reloading one. The 


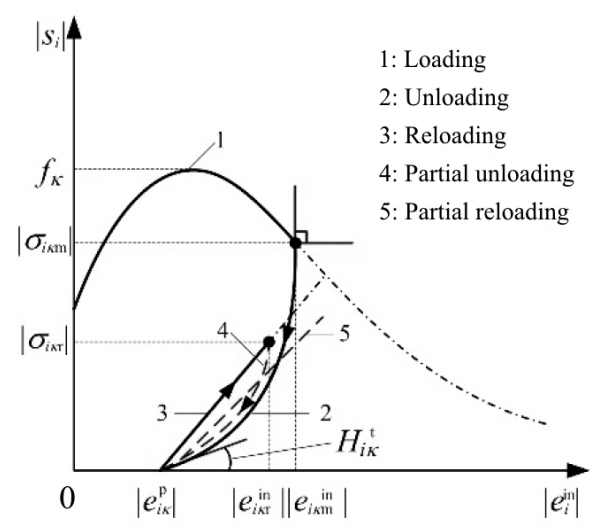

Unloading branch

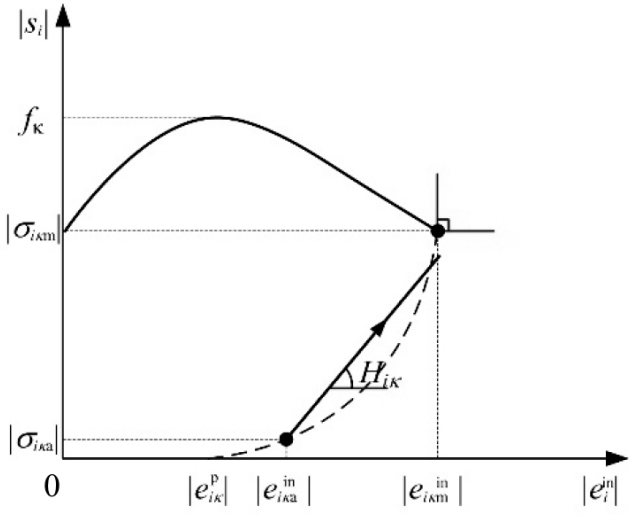

Reloading branch

Fig. 1. The definition of unloading and reloading paths subjected to cyclic loading.

loading path is defined by the yielding function, given in Eq. (4), considering the effect of stress state on the material strength:

$$
\begin{aligned}
& F=\frac{1}{1-\alpha}\left(q-3 \alpha p+\beta\left\langle\sigma_{\max }\right\rangle\right)-\bar{\sigma}_{c}\left(\varepsilon_{c}^{i n}\right)=0, \\
& \alpha=\frac{\left(f_{b c}-f_{c}\right)}{\left(2 f_{b c}-f_{c}\right)}, \quad \beta=\frac{\bar{\sigma}_{c}\left(\varepsilon_{c}^{i n}\right)}{\bar{\sigma}_{t}\left(\varepsilon_{t}^{i n}\right)}(1-\alpha)-(1+\alpha), \\
& p=-\frac{1}{3} \sigma_{i i}, \quad q=\sqrt{\frac{3}{2} s_{i j} s_{i j}}, \quad s_{i j}=\sigma_{i j}+p,
\end{aligned}
$$

in which $p$ denotes the hydrostatic pressure, $q$ stands for the Mises stress, $\alpha$ and $\beta$ are dimensionless parameters, $f_{b c}$ and $f_{c}$ represent the maximum compressive stresses under equibiaxial and uniaxial compression, respectively, and $\bar{\sigma}_{c}\left(\varepsilon_{c}^{i n}\right)$ and $\bar{\sigma}_{t}\left(\varepsilon_{t}^{i n}\right)$ denote the equivalent stresses corresponding to uniaxial compression and tension. The symbol $\langle\cdot\rangle$ is the McCauley bracket which means $\langle x\rangle=\frac{1}{2}(x+|x|)$.

The hysteretic behavior is approximately modeled by employing the nonlinear unloading path and linear reloading one. Figure 1 shows the unloading/reloading paths defined regarding stress and inelastic strain. The nonlinear unloading path is given in Eq. (5):

$$
\begin{aligned}
& \left|s_{i}\right|=\left|\sigma_{i \kappa m}\right|+B^{\kappa}\left|e_{i \kappa m}^{i n}-e_{i}^{i n}\right|+C^{\kappa}\left|e_{i \kappa m}^{i n}-e_{i}^{i n}\right|^{\alpha^{\kappa}}, \\
& B^{\kappa}=\beta^{\kappa}\left[\alpha^{\kappa}\left|\sigma_{i \kappa m}\right|\left|e_{i \kappa m}^{i n}-e_{i \kappa}^{p}\right|^{\alpha^{\kappa}-1}-H_{i \kappa}^{t}\left|e_{i \kappa m}^{i n}-e_{i \kappa}^{p}\right|\right], \\
& C^{\kappa}=\beta^{\kappa}\left[H_{i \kappa}^{t}\left|e_{i \kappa m}^{i n}-e_{i \kappa}^{p}\right|-\left|\sigma_{i \kappa m}\right|\right], \\
& \beta^{\kappa}=\frac{1}{1-\alpha^{\kappa}}\left|e_{i \kappa m}^{i n}-e_{i \kappa}^{p}\right|^{-\alpha^{\kappa}},
\end{aligned}
$$

where $i$ denotes the $i$ th damage axis and $\kappa \in(t, c)$ represents tension or compression, $s_{i}$ and $e_{i}^{i n}$ are the present principal stress and inelastic strain, $\sigma_{i \kappa m}$ and $e_{i \kappa m}^{i n}$ denote the stress and inelastic strain at the start of unloading, $e_{i \kappa}^{p}$ is the plastic strain, $H_{i \kappa}^{t}$ denotes 
the tangent modulus of unloading path corresponding to zero stress, and $\alpha^{\kappa} \in(0,1)$ is a constant.

Besides, the reloading path is defined by Eq. (6) as a linear path

$$
\begin{aligned}
& \left|s_{i}\right|=\left|\sigma_{i \kappa a}+H_{i \kappa}\right| e_{i}^{i n}-e_{i \kappa a}^{i n} \mid, \\
& H_{i \kappa}=\frac{1-d_{i}}{d_{i}} E, \quad d_{i} \in[0,1],
\end{aligned}
$$

in which $\sigma_{i \kappa a}$ and $e_{i \kappa a}^{i n}$ are the stress and inelastic strain at the start of the reloading path, and $H_{i \kappa}$ is the modulus determined by the damage factor $d_{i}$. Herein, $d_{i}$ is defined concerning tensile and compressive damage variables, which can be referred to reference [16]. The definitions of partial unloading and partial reloading are similar to those of unloading and reloading and also can be referred to [16].

2. A Numerical Example of a Notched Beam Test. This model is used to simulate the damaging process of plain concrete beam tests conducted by Hordijk [19]. As shown in Fig. 2, square-cross-section beams, whose dimensions were $500 \times 100 \times 50 \mathrm{~mm}$, had an initial notch depth $\delta=10,30$, and $50 \mathrm{~mm}$, respectively, and were subjected to four-point loading. The material properties used herein are as follows: elastic modulus $E_{0}=3.8 \cdot 10^{4}$ $\mathrm{MPa}$, Poisson's ratio $v=0.2$, tensile strength $f_{t}=3.0 \mathrm{MPa}$, tensile fracture energy density $G_{t}^{f}=125 \mathrm{~N} / \mathrm{m}$. The details of Hordijk's experiments can be referred to study [19]. Numerical analyses are carried out by the finite element analysis program ABAQUS since this model has been embedded into ABAQUS by its user-defined subroutine UMAT. Four-point plane stress element with dimensions $5 \times 5 \mathrm{~mm}$ is utilized to model those concrete beams in the numerical simulations.

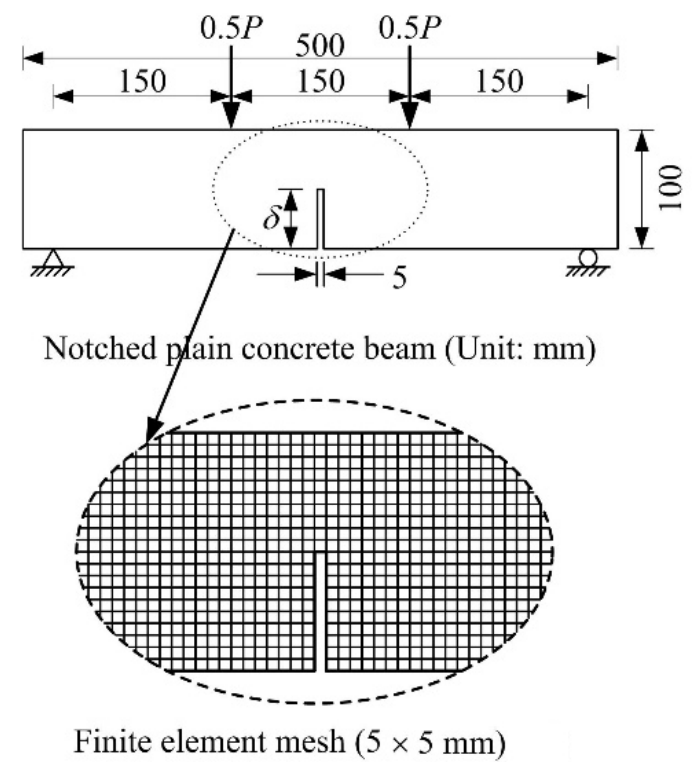

Fig. 2. Testing plan and finite element mesh of notched beam test.

2.1. Monotonic Loading Tests. The stress-inelastic strain curve is an important effect on numerical simulation. Four types of the strain-softening curve are used in the monotonic loading analyses, as illustrated in Fig. 3. The definitions of these curves are as follows: 


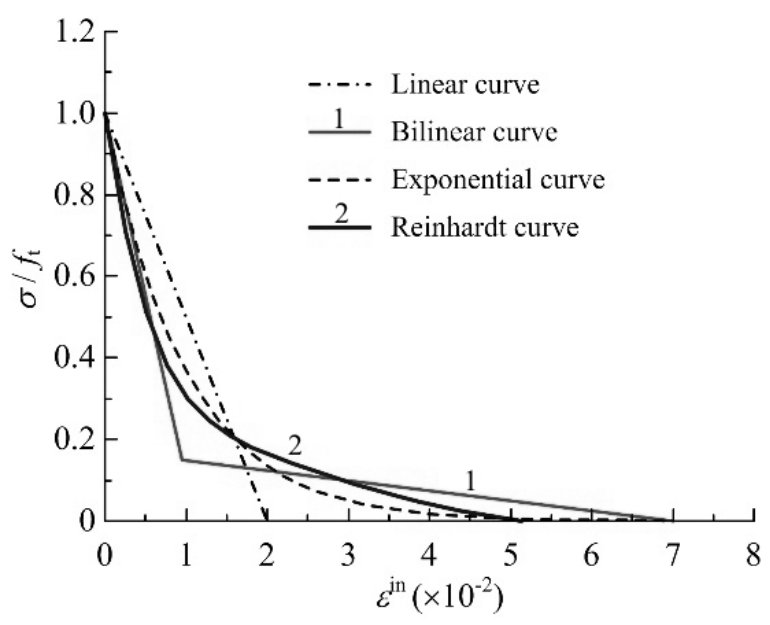

Fig. 3. Tensile strain softening curves of concrete.

(1) The linear softening curve is given by Eq. (7):

$$
\frac{\sigma}{f_{t}}=1-\frac{\varepsilon^{i n}}{\varepsilon_{f}}, \quad \varepsilon_{f}=\frac{2 G_{t}^{f}}{f_{t} h},
$$

where $\sigma$ and $\varepsilon^{i n}$ are the stress and inelastic strain, and $G_{t}^{f}, f_{t}$, and $h$ denote the tensile fracture energy density, tensile strength, and characteristic size of the damaged element, respectively.

(2) The bilinear softening curve is defined by Eq. (8) is proposed in [20]:

$$
\frac{\sigma}{f_{t}}=\left\{\begin{array}{ll}
1-0.85 \frac{\varepsilon^{i n}}{\varepsilon_{f}}, & 0 \leq \varepsilon^{i n} \leq \varepsilon_{1}, \\
0.15 \frac{\varepsilon_{f}-\varepsilon^{i n}}{\varepsilon_{f}-\varepsilon_{1}}, & \varepsilon_{1}<\varepsilon^{i n} \leq \varepsilon_{f},
\end{array} \quad \varepsilon_{1}=2 \frac{G_{t}^{f}}{f_{t} h}-0.15 \varepsilon_{f}, \quad \varepsilon_{f}=\frac{\alpha_{F} G_{t}^{f}}{f_{t} h},\right.
$$

where it is assumed that the maximum diameter of aggregate is $16 \mathrm{~mm}$ and $\alpha_{F}=7$ is given.

(3) The exponent one proposed by Karihaloo [21] is given as follows:

$$
\frac{\sigma}{f_{t}}=\exp \left(-0.405 \frac{\varepsilon^{i n}}{\varepsilon_{0}}\right), \quad \varepsilon_{0}=6.405 \frac{G_{t}^{f}}{f_{t} h} .
$$

(4) The Reinhardt's one [22] is defined as

$$
\frac{\sigma}{f_{t}}=\left[1+\left(c_{1} \frac{\varepsilon^{i n}}{\varepsilon_{f}}\right)^{3}\right] \exp \left(-c_{2} \frac{\varepsilon^{i n}}{\varepsilon_{f}}\right)-e^{-c_{2}}\left(1+c_{1}^{3}\right) \frac{\varepsilon^{i n}}{\varepsilon_{f}},
$$

in which $c_{1}=3$ and $c_{2}=6.93$ are used and, therefore, $\varepsilon_{f}=5.136 G_{t}^{f} / f_{t} h$. 
$\mathrm{T}$ a b 1 e 1

The Comparison of the Calculated Peak Value of Load $P_{\max }$ and Experimental One $P_{e \max }[19]$

\begin{tabular}{|c|c|c|c|c|c|c|c|c|c||}
\hline \multirow{2}{*}{$\begin{array}{c}\text { Notch } \\
\text { depth } \\
\delta, \mathrm{mm}\end{array}$} & \begin{tabular}{c} 
Experimental \\
\cline { 2 - 11 }
\end{tabular} & \multicolumn{2}{|c|}{ Linear } & \multicolumn{2}{|c|}{ Bilinear } & \multicolumn{2}{|c|}{ Exponential } & \multicolumn{2}{|c|}{ Reinhardt's } \\
\hline 10 & 3.62 & $\begin{array}{c}P_{\max }, \\
\mathrm{kN}\end{array}$ & $\begin{array}{c}\Delta, \\
\%\end{array}$ & $\begin{array}{c}P_{\max }, \\
\mathrm{kN}\end{array}$ & $\begin{array}{c}\Delta, \\
\%\end{array}$ & $\begin{array}{c}P_{\max }, \\
\mathrm{kN}\end{array}$ & $\begin{array}{c}\Delta, \\
\%\end{array}$ & $\begin{array}{c}P_{\max }, \\
\mathrm{kN}\end{array}$ & $\begin{array}{c}\Delta, \\
\%\end{array}$ \\
\hline 30 & 2.53 & 2.93 & 15.8 & 2.61 & 3.2 & 2.65 & 4.7 & 2.51 & -0.8 \\
\hline 50 & 1.42 & 1.59 & 12.0 & 1.43 & 0.7 & 1.44 & 1.4 & 1.37 & -3.5 \\
\hline
\end{tabular}

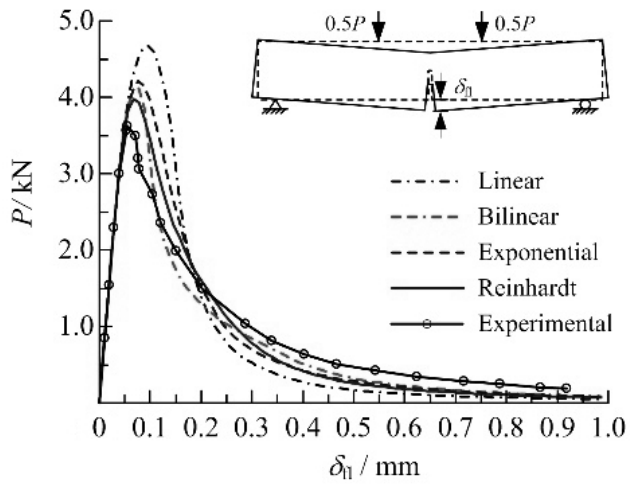

notched depth $\delta=10 \mathrm{~mm}$

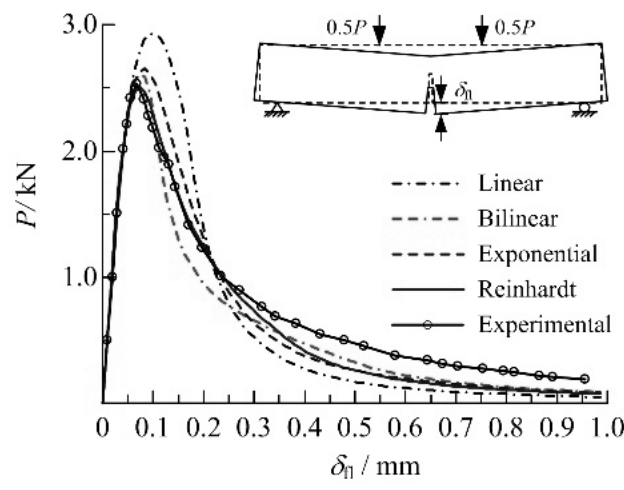

notched depth $\delta=30 \mathrm{~mm}$

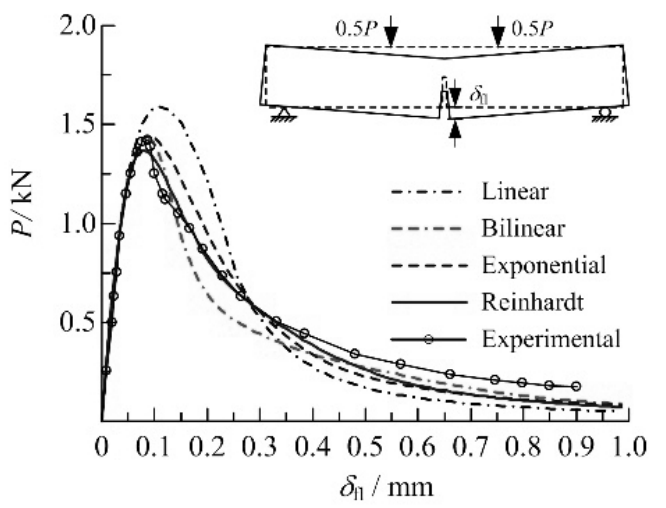

notched depth $\delta=50 \mathrm{~mm}$

Fig. 4. The $P-\delta_{f l}$ curves of concrete beams subjected to monotonic loading (compared with experimental results [19]).

Figure 4 shows the responses between load and deflection, i.e., the $P-\delta_{f l}$ curves, compared to the experimental ones. The peak value of load in each case is given in Table 1, where $\Delta=\left(P_{e \max }-P_{\max }\right) / P_{e \max }$ denotes the error between the numerical result and the experimental one. As illustrated in Fig. 4, softening curves have a significant influence on the structural responses of concrete beams although the calculated $P-\delta_{f l}$ curves are comparable to the experimental ones. In the case with the linear softening curve, numerical responses are significantly different from experimental ones, as they overestimate the values of peak load from 12 to $29 \%$ and their post-peak curves are much steeper than the 
experimental ones. When utilizing bilinear and exponential softening curves, the numerical $P-\delta_{f l}$ curves are much closer to the experimental results than those obtained by the linear softening curve. Furthermore, the numerical responses in the case with the Reinhardt's curve are in the closest agreement with experimental ones, in which the errors of peak load are from -3.5 to $9.9 \%$. Consequently, the Reinhardt's curve best reflects the influence of strain-softening effect on the structural response of the notched concrete beams.

2.2. Cyclic Loading Tests. Cyclic tests of the notched beams are also modeled by the anisotropic damage model. The loading path is determined by the Reinhardt curve, and the unloading and reloading ones are defined by Eqs. (5) and (6). Equation (11) gives the plastic strain $e_{i t}^{p}$ and tangent modulus $H_{i t}^{t}$ at a zero stress:

$$
e_{i t}^{p}=c_{t}+k_{t} e_{i}^{i n}, \quad H_{i t}^{t}=E_{0} A_{t} \exp \left[B_{t}\left(\frac{e_{i}^{i n}}{\varepsilon_{0}}\right)\right],
$$

where $c_{t}=0, k_{t}=0.4, A_{t}=0.5, B_{t}=1.0$, and $\varepsilon_{0}=f_{t} / E_{0}$. The exponential constant $\alpha_{\kappa}$ defining the unloading curve is given as 0.5 .

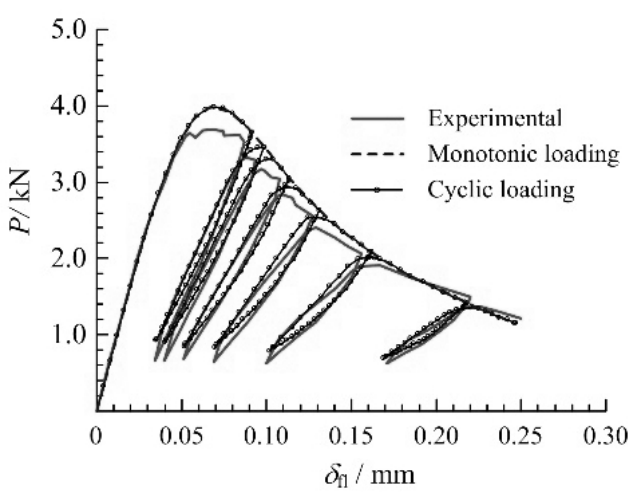

notched depth $\delta=10 \mathrm{~mm}$

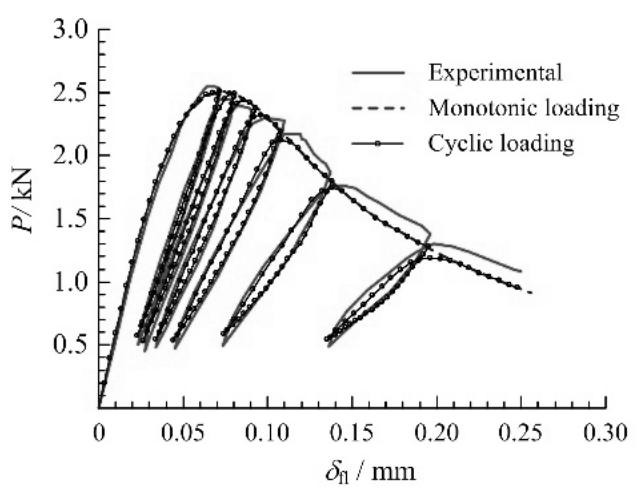

notched depth $\delta=30 \mathrm{~mm}$

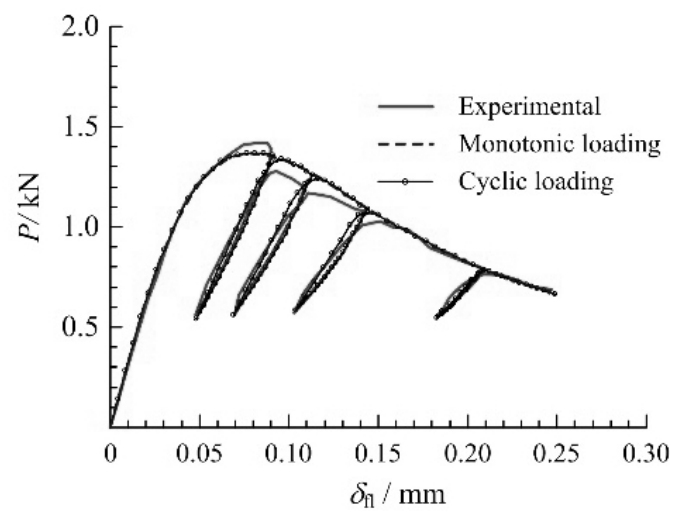

notched depth $\delta=50 \mathrm{~mm}$

Fig. 5. The $P-\delta_{f l}$ curves of concrete beams subjected to cyclic loading [19].

Figure 5 shows the $P-\delta_{f l}$ curves of notched beams obtained by numerical simulation, subjected to monotonic and cyclic loading, compared to the experimental results. The $P-\delta_{f l}$ curves under monotonic loading are identical to the envelopes of 
those under cyclic loading because they are determined by the loading path that gives the boundary of structural responses of concrete beams. The calculated results are highly consistent with experimental ones, which indicates that this anisotropic damage model reasonably reflects the stiffness degradation and hysteretic behavior of concrete. Slight difference between numerical and experimental results, such as the peak load, the post-peak envelope, and the unloading/reloading paths, results from the fact that the envelopes of the numerical responses are different from those of experiments. The hysteretic behavior in the material level is approximately modeled by employing the nonlinear unloading and linear reloading paths, which obtains a reasonably hysteretic response in the structural level. Therefore, this method applies to modeling the effect of hysteretic behavior on the structural response of concrete structures.

Conclusions. The fracture behavior of concrete beams was investigated by an anisotropic damage model. The results draw the following conclusions:

1. The strain softening curves significantly affect the numerical response of concrete beams. The linear softening curve overestimates the peak value of the load and causes steeper post-peak responses than experimental ones while the Reinhardt's one obtains the closest responses compared with experimental ones.

2. The method of nonlinear unloading/linear reloading applies to represent the hysteretic behavior of concrete. Using this method, the calculated load-deflection responses agree well with experimental ones. The numerical results make it possible to conclude that the stiffness degradation and hysteretic behavior can be well represented by the present anisotropic damage model.

Acknowledgments. The present work has been supported by the National Natural Science Foundation of China, with Grant No. 51578088. The authors are grateful for this support.

1. J. Mazars and G. Pijaudier-Cabot, "Continuum damage theory - application to concrete," J. Eng. Mech., 115, No. 2, 345-365 (1989).

2. J. Mazars, Y. Berthaud, and S. Ramtani, "The unilateral behavior of damaged concrete," Eng. Fract. Mech., 35, 629-635 (1990).

3. E. Papa, "A damage model for concrete subjected to fatigue loading," Eur. J. Mech. A-Solids, 12, No. 3, 429-440 (1993).

4. M. Cervera, J. Oliver, and O. Manzoli, "A rate-dependent isotropic damage model for the seismic analysis of concrete dams," Earth. Eng. Struct. D., 25, No. 9, 987-1010 (1996).

5. B. Richard, F. Ragueneau, C. Cremona, and L. Adelaide, "Isotropic continuum damage mechanics for concrete under cyclic loading: Stiffness recovery, inelastic strains, and frictional sliding," Eng. Fract. Mech., 77, No. 8, 1203-1223 (2010).

6. U. Haussler-Combe and T. Kuhn, "Modeling of strain rate effects for concrete with viscoelasticity and retarded damage," Int. J. Impact Eng., 50, 17-28 (2012).

7. C. Desprez, J. Mazars, P. Kotronis and P. Paultre, "Damage model for FRP-confined concrete columns under cyclic loading," Eng. Struct., 48, No. 3, 519-531 (2013).

8. E. Papa and A. Taliercio, "Anisotropic damage model for the multiaxial static and fatigue behaviour of plain concrete," Eng. Fract. Mech., 55, No. 2, 163-179 (1996).

9. A. Alliche, "Damage model for fatigue loading of concrete," Int. J. Fatigue, 26, No. 9, 915-921 (2004).

10. P. Lu, Q. Li, and Y. Song, "Damage constitutive of concrete under uniaxial alternate tension-compression fatigue loading based on double bounding surfaces," Int. J. Solids Struct., 41, No. 11, 3151-3166 (2004). 
11. P. Badel, V. Godard, and J. B. Leblond, "Application of some anisotropic damage model to the prediction of the failure of some complex industrial concrete structures," Int. J. Solids Struct., 44, No. 18, 5848-5874 (2007).

12. M. L. M. François, "A damage model based on Kelvin eigentensors and Curie principle," Mech. Mater., 44, 23-34 (2012).

13. J. Y. Wu and S. L. Xu, "Reconsideration on the elastic damage/degradation theory for the modeling of microcrack closure-reopening (MCR) effects," Int. J. Solids Struct., 50, No. 5, 795-805 (2013).

14. M. Ngo, A. Ibrahimbegovic, and D. Patherie, "Continuum damage model for thermo-mechanical coupling in quasi-brittle materials," Eng. Struct., 50, No. 3, 170-178 (2013).

15. M. A. Hariri-Ardebili and H. Mirzabozorg, "Orthotropic material and anisotropic damage mechanics approach for numerically seismic assessment of arch damreservoir-foundation system," Strength Mater., 45, No. 6, 648-665 (2013).

16. Y. C. Long and Y. M. He, "An anisotropic damage model for concrete structures under cyclic loading-uniaxial modeling," J. Phys. Conf. Ser., 842, No. 1, 012040 (2017).

17. ABAQUS Analysis User's Guide, Dassault Systemes, Velizy-Villacoublay Cedex, France (2016).

18. ABAQUS User Subroutines Reference Guide, Dassault Systemes, Velizy-Villacoublay Cedex, France (2016).

19. D. A. Hordijk, Local Approach to Fatigue of Concrete, Doctor's Thesis, Technische Universiteit Delft, Delft, Netherlands (1991).

20. CEB-FIP Model Code 1990: Design Code, Comite Euro-International Du Beton, Thomas Telford Services Ltd, London (1993).

21. B. L. Karihaloo, Fracture Mechanics and Structural Concrete, Longman Scientific \& Technical, Harlow, UK (1995).

22. H. W. Reinhardt, "Fracture mechanics of an elastic softening material like concrete," Heron, 29, No. 2, 1-42 (1984). 